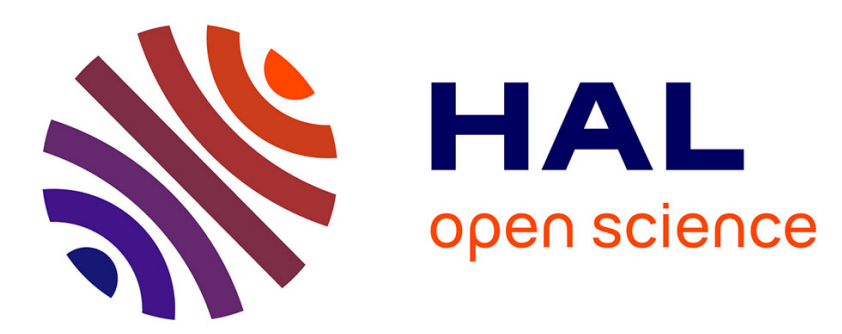

\title{
Repetitive Control Design Based on Forwarding for Nonlinear Minimum-Phase Systems
}

\author{
Daniele Astolfi, Swann Marx, Nathan van de Wouw
}

\section{To cite this version:}

Daniele Astolfi, Swann Marx, Nathan van de Wouw. Repetitive Control Design Based on Forwarding for Nonlinear Minimum-Phase Systems. Automatica, In press. hal-03157572

\author{
HAL Id: hal-03157572 \\ https://hal.science/hal-03157572
}

Submitted on 3 Mar 2021

HAL is a multi-disciplinary open access archive for the deposit and dissemination of scientific research documents, whether they are published or not. The documents may come from teaching and research institutions in France or abroad, or from public or private research centers.
L'archive ouverte pluridisciplinaire HAL, est destinée au dépôt et à la diffusion de documents scientifiques de niveau recherche, publiés ou non, émanant des établissements d'enseignement et de recherche français ou étrangers, des laboratoires publics ou privés. 


\title{
Repetitive Control Design Based on Forwarding for Nonlinear Minimum-Phase Systems
}

\author{
Daniele Astolfi ${ }^{\mathrm{a}}$, Swann Marx ${ }^{\mathrm{b}}$, Nathan van de Wouw ${ }^{\mathrm{c}, \mathrm{d}}$ \\ ${ }^{a}$ Univ Lyon, Université Claude Bernard Lyon 1, CNRS, LAGEPP UMR 5007, 43 boulevard du 11 novembre 1918, F-69100, Villeurbanne, \\ France. \\ ${ }^{b}$ LS2N, École Centrale de Nantes \& CNRS UMR 6004, F-44000 Nantes, France. \\ ${ }^{c}$ Department of Mechanical Engineering, Eindhoven University of Technology, 5600 Eindhoven, Netherlands \\ ${ }^{d}$ Department of Civil, Environmental and Geo-Engineering, University of Minnesota, Minneapolis, MN 55455-0116 USA
}

\begin{abstract}
We propose a new design for a repetitive control scheme for nonlinear minimum-phase systems with arbitrary relative degree and globally Lipschitz nonlinearities. We represent the delay of the repetitive control scheme as a transport equation and we propose a new forwarding-based (partial) state-feedback design that uses not only the boundary information of the delay, but the entire state of the transport equation representing the delay. Through a rigorous mathematical analysis, we show that, from a theoretical point of view, asymptotic convergence of the desired regulated output can be achieved with the proposed control design.
\end{abstract}

\section{Introduction}

Repetitive Control (RC) techniques for continuous-time systems have been developed at the end of the 80's for tracking and/or rejecting periodic signals of known pe$\operatorname{riod} T$, see [1]. Supported by the well-known "internal model principle" (see, e.g., [2]) stating that a robust controller needs to incorporate a copy of the model generating the references/disturbances, the main idea of $\mathrm{RC}$ relies on the use of a delay as universal generator of periodic signals. Such a delay (of time $T$ ) is then embedded in the feedback regulator in order to achieve asymptotic regulation of the desired output. As a consequence, one of the main difficulties in RC is to guarantee the stability of a closed-loop system incorporating a delay, i.e., being an infinite-dimensional system. The aforementioned technical issues and the fact that, from a practical point of view, the implementation of infinite-dimensional control solutions is considered as challenging, widely justified the research of finite-dimensional versions of RC schemes. Indeed, after the seminal work of [3], a large part of the RC-community approached the problem in the discrete-time domain, see for instance, [4-7]. This partial change of paradigm made also a step towards computational techniques such as it-

\footnotetext{
This research was partially supported by the French Grant ANR ODISSE (ANR-19-CE48-0004-01) and was also conducted in the framework of the regional programme "Atlanstic 2020, Research, Education and Innovation in Pays de la Loire", supported by the French Region Pays de la Loire and the European Regional Development Fund.

Email addresses: daniele.astolfi@univ-lyon1.fr (Daniele Astolfi), swann.marx@ls2n.fr (Swann Marx), n.v.d.wouw@tue.nl (Nathan van de Wouw )
}

erative learning control and run-to-run control, see, e.g., [8-10].

Despite the large progress in practical applications (see, among them, [11-14]), when considering the problem of a RC-design in the continuous-time domain, many questions are still open. In particular, it is not clear whether an exact (i.e., infinite-dimensional) RC-scheme can be used (from a theoretical point of view) to achieve asymptotic convergence of the desired regulated output. In fact, one of the main limitations of existing continuous-time schemes is that only "boundary information" (namely, the values of the delayed signal at instants $t$ and $t+T$ ) is used for control purposes. Such a constraint, however, strongly restricts the class of systems to which a RC-scheme can be applied, that is nonlinear systems which are strictly input passive (in other words, with a direct feedthrough term). We refer to [1] for a proof for linear systems where it is shown that exponential stability of a (continuous-time) linear system incorporating a pure delay in the RC-scheme can be achieved only for systems having zero-relative degree between the input and the regulated output; alternatively, see $[15,16]$ for a proof in the context of nonlinear systems based on dissipativity operators.

Somehow, to our point of view, the observation in [1, Proposition 2] limited the developments of infinitedimensional continuous-time research in $\mathrm{RC}$, since mostly finite-dimensional approximations have been proposed after this seminal work. Among them, we recall the use of low-pass filters in $[17,18]$, and the use of an approximated harmonic representation of the delay via Fourier series, see, e.g., [14, 19]. Note that equivalent designs involving the use of such a harmonic representation have been also studied in output regulation literature, see, e.g., [20-24] 
and references therein.

The objective of this work is therefore to study the problem of designing an exact RC-scheme (that is, an infinitedimensional one) for nonlinear systems with global convergence properties. To the best of the authors' knowledge, this problem is still open. We focus in particular on systems which are minimum-phase, with a global, well defined $^{1}$ arbitrary relative degree and globally Lipschitz nonlinearities and we propose a new approach for the RCfeedback design. For this, we follow the idea of using an "in-domain feedback" (that is, all the values of the delayed signals between $t$ and $t+T$ ) instead of a pure "boundary feedback" (that is, only the values at $t$ and $t+T)$. Although such an idea is not new in discretetime RC-schemes (see, e.g., [3, 6, 10]), to the best of the authors' knowledge, it has never been investigated in the literature of continuous-time RC-schemes. In order to construct the feedback, differently from classical approaches, we represent the delay as a transport equation ${ }^{2}$, resulting in an overall system which is in the so-called "feedforward-form" [26]. Then, inspired by the "forwarding approach" (see [23, 26] and references therein) we construct a new (partial) state feedback law, and we prove that the proposed dynamical regulator satisfies the internal model property, namely it can reproduce any periodic trajectory. We also show that the proposed control law can be equivalently written in the pure time-domain without the use of the transport equation. The latter, however, is essential for the overall analysis: indeed, given to the infinite-dimensional properties of the RC-scheme, well-posedness, regularity and stability of the solutions of the resulting closed-loop system are rigorously addressed. Global asymptotic stability of the regulated output to the desired periodic reference is finally proved.

With respect to existing literature of continuous-time RC-schemes, our result is completely new as it certificates the existence with a constructive design of an infinitedimensional RC-design achieving exact asymptotic stability, for nonlinear systems with arbitrarily relative degree. The design of the proposed forwarding-control law is inspired by our recent preliminary work [27] in the context of stabilization of a partial differential equation equation coupled with a linear scalar ordinary differential equation. The extension to the proposed nonlinear paradigm is however non-trivial.

This work is organized as follows. First, in Section 2 we detail the problem statement. The main result concerning the design of a RC-scheme for minimum-phase nonlinear systems with unitary relative degree is presented in Section 3. Next, using the results in Section 3 as a stepping stone, in Section 4, we address the case of systems having a relative degree larger than one. Illustrative examples are provided in Section 5. Conclusions are derived in Sec-

\footnotetext{
${ }^{1}$ In the sense of [25, Chapter 4].

${ }^{2}$ The use of a transport-equation representation for the delay has been recently used in $[15,16]$ but for analysis purposes only.
}

tion 6. Proofs are postponed to the Appendix.

Notation. We denote with $\mathbb{R}$ the space of real numbers and $\mathbb{C}$ the space of complex numbers. Set $\mathbb{R}_{+}:=[0, \infty)$. For a function $w:(t, x) \in \mathbb{R}_{+} \times[0,1] \mapsto w(t, x) \in \mathbb{R}$, the notation $w_{t}$ (respectively, $w_{x}$ ) denotes the partial derivative of $w$ with respect to the variable $t$ (respectively, with respect to the variable $x$ ). We keep the notation for the weak and the strong definition of partial derivatives. When a function $w$ depends only on the variable of the time $t$ (respectively, space $x$ ), we denote its derivative by $\dot{w}$ (respectively, $w^{\prime}$ ). Let $L^{2}(0,1)$ be the Hilbert space of real-valued squareintegrable functions over the interval $(0,1)$. We denote $\langle f, g\rangle_{L^{2}}=\int_{0}^{1} f g$ the inner product between $f, g \in L^{2}(0,1)$ and $\|f\|_{L^{2}}$ the induced norm. Let $H^{1}(0,1) \subset L^{2}(0,1)$ be the Hilbert space of real-valued absolutely continuous functions over $[0,1]$ with square-integrable derivative. We denote $\langle f, g\rangle_{H^{1}}=\langle f, g\rangle_{L^{2}}+\left\langle f^{\prime}, g^{\prime}\right\rangle_{L^{2}}$ the inner product between $f, g \in H^{1}(0,1)$ and $\|f\|_{H^{1}}$ the induced norm.

\section{Problem Statement for Nonlinear Repetitive Control}

\subsection{Repetitive Control Scheme Representation}

Any periodic signal of period $T$ can be generated by the time-delay system represented in Figure 1a and described by the following transfer function (where $s \in \mathbb{C}$ represents the Laplace variable)

$$
R(s)=\frac{\exp (-T s)}{1-\exp (-T s)},
$$

see for instance [1]. Hence, in order to solve the problem of reference tracking of disturbance rejection of periodic signals, it is reasonable to include such a delay model in the regulator, as expected from the internal model principle stated in [2]. Regulators following this paradigm are usually denoted in literature as Repetitive Control (RC), see [1].

In this work, we represent the delay $\exp (-T s)$ of the transfer function (1), by means of the (alternative but equivalent) following transport equation representation:

$$
\begin{aligned}
\eta_{t}(t, x) & =-\frac{1}{T} \eta_{x}(t, x) & & \forall(t, x) \in \mathbb{R}_{+} \times[0,1], \\
\eta(t, 0) & =p(t) & & \forall t \in \mathbb{R}_{+}, \\
\eta(0, x) & =0 & & \forall x \in[0,1],
\end{aligned}
$$

where $\eta(t, x)$ is a state variable taking values in $\mathbb{R}$ for each $(t, x) \in \mathbb{R}_{+} \times[0,1]$, and $p$ is the source of the delay equation. It is possible to see that the output $\eta(t, 1)$ of system (2) is equal to the delayed version of the source input $p(t)$ at any $t \geq T$. Indeed, recall that, as shown in [28, Chapter $2.1]$, the general solution to (2) is given by

$$
\eta\left(t+\left(x-x^{\prime}\right) T, x\right)=\eta\left(t, x^{\prime}\right)
$$

for all $t \in \mathbb{R}_{+}$and for all $0 \leq x^{\prime} \leq x \leq 1$. Therefore, by using (3) with the boundary conditions in (2), we obtain

$$
\eta(t+T, 1)=\eta(t, 0)=p(t), \quad \forall t \in \mathbb{R}_{+} .
$$




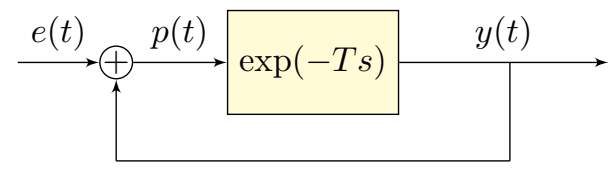

(a) Delay representation.

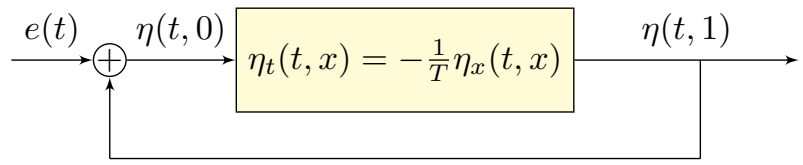

(b) Transport-equation representation.

Figure 1: Equivalent representations of an internal-model designs in RC-schemes.

As a consequence, the input-output relation of a standard RC-scheme (1) represented in Figure 1a, which can be computed as

$$
y(t)=\mathcal{L}^{-1}\{\exp (-s T) \mathcal{L}\{p(t)\}\}, \quad p(t)=e(t)+y(t),
$$

where we denoted with $\mathcal{L}$ the Laplace transform and by $\mathcal{L}^{-1}$ its inverse, can be equivalently described, by using the delay representation given by (2), as

$$
\begin{aligned}
\eta_{t}(t, x) & =-\frac{1}{T} \eta_{x}(t, x) & & \forall(t, x) \in \mathbb{R}_{+} \times[0,1], \\
\eta(t, 0) & =p(t) & & \forall t \in \mathbb{R}_{+}, \\
\eta(0, x) & =0 & & \forall x \in[0,1], \\
p(t) & =e(t)+y(t) & & \forall t \in \mathbb{R}_{+}, \\
y(t) & =\eta(t, 1) & & \forall t \in \mathbb{R}_{+},
\end{aligned}
$$

as depicted in Figure 1b. Note that, from a conceptual point of view, the representation (4) can be interpreted as the continuous-time and continuous-space version of the discrete RC-scheme proposed in [3], in which the transport is represented, in the discrete-time domain, as a chain of $N$ delays discrete operators $z^{-1}$. In particular, compare Figure $1 \mathrm{~b}$ to [3, Fig. 2].

The objective of this work is therefore to develop a new RC-feedback design using the representation (4). Note that, differently from classical schemes, we will allow us to use not only the information of the delay at the boundaries $\eta(t, 0)$ and $\eta(t, 1)$, but also the full-state information of $\eta(t, x)$ at all $x \in[0,1]$. Such a choice, which is, to the best of authors' knowledge, not exploited in other works concerning continuous-time RC-schemes, gives us a larger degree of freedom in the feedback design, allowing to achieve asymptotic stability of the overall closed-loop system for a system of arbitrarily large relative degree.

Implementation aspects related to the discretization of the proposed representation will not be discussed in this brief article and will be addressed in future works.

\subsection{Problem Statement}

In this work, we consider the problem of designing a RCscheme for minimum-phase nonlinear systems that can be written, possibly after a change of coordinates, in the form:

$$
\begin{aligned}
\dot{z} & =f(t, z, e) \\
\dot{e} & =q(t, z, e)+u,
\end{aligned}
$$

where $(z, e) \in \mathbb{R}^{r} \times \mathbb{R}$ is the system state, with the $z$ dynamics being the so-called zero-dynamics, $e \in \mathbb{R}$ is the regulated output, and $u \in \mathbb{R}$ is the control input. The more general case of higher relative degree will be discussed in Section 4, since, by using a standard change of coordinates, the higher relative degree case can be reduced to the unitary one (see, for instance, [24]).

The following assumptions on the functions $f, q$ in (5) are adopted throughout this paper.

Assumption 1. The functions $f, q: \mathbb{R} \times \mathbb{R}^{n} \times \mathbb{R}$ are globally Lipschitz, $C^{2}$ in all their arguments and periodic with respect to the first argument. In particular, there exists $\ell_{f}, \ell_{q}>0$ such that

$$
\begin{gathered}
|f(t, z, e)-f(t, \hat{z}, \hat{e})| \leq \ell_{f}|z-\hat{z}|+\ell_{f}|e-\hat{e}|, \\
|q(t, z, e)-q(t, \hat{z}, \hat{e})| \leq \ell_{q}|z-\hat{z}|+\ell_{q}|e-\hat{e}|,
\end{gathered}
$$

for all $t \in \mathbb{R}_{+}, z, \hat{z} \in \mathbb{R}^{n}$ and $e, \hat{e} \in \mathbb{R}$, and there exists $T>0$ such that

$$
f(t+T, z, e)=f(t, z, e), \quad q(t+T, z, e)=q(t, z, e),
$$

for all $t \in \mathbb{R}_{+}$and $(z, e) \in \mathbb{R}^{n} \times \mathbb{R}$.

Assumption 2. The zero-dynamics $\dot{z}=f(t, z, 0)$ admits a unique $C^{2}$ T-periodic bounded solution $\bar{z}(t)$ which is globally uniformly stable ${ }^{3}$. In particular, there exists a positive definite function $V: \mathbb{R} \times \mathbb{R}^{n} \rightarrow \mathbb{R}_{+}$and class $\mathcal{K}_{\infty}$ functions $^{4} \underline{\alpha}, \bar{\alpha}$ and real numbers $\alpha, \gamma>0$ satisfying

$$
\underline{\alpha}(|z-\bar{z}(t)|) \leq V(t, z-\bar{z}(t)) \leq \bar{\alpha}(|z-\bar{z}(t)|)
$$

for all $(t, z) \in \mathbb{R}_{+} \times \mathbb{R}^{n}$ and

$$
\begin{aligned}
\langle\nabla V(t, z-\bar{z}(t)), f(t, z, e)- & f(t, \bar{z}(t), 0)\rangle \leq \\
& -\alpha|z-\bar{z}(t)|^{2}+\gamma|e|^{2}
\end{aligned}
$$

for $\operatorname{all}(t, z, e) \in \mathbb{R}_{+} \times \mathbb{R}^{n} \times \mathbb{R}$.

Remark. (About Assumptions 1 and 2) Assumption 1 states that all functions characterizing the vector field of (5) are globally Lipschitz, while Assumption 2 states that the unperturbed zero-dynamics of system (5) possesses an attractive steady-state which is globally asymptotically stable and that the z-dynamics with nonzero $e(t)$ is input-to-state stable with respect to $e$.

Note that Assumption 2 is rather standard in the context of global stabilization (in which $\bar{z}(t)=0$ and $q(t, \bar{z}(t), 0)=$

\footnotetext{
${ }^{3}$ See $[29$, Definition 4.4]

${ }^{4}$ A function $\alpha: \mathbb{R}_{+} \rightarrow \mathbb{R}_{+}$is said to be of class $\mathcal{K}$ if $\alpha$ is continuous, increasing, and $\alpha(0)=0$. If moreover $\lim _{x \rightarrow+\infty} \alpha(x)=+\infty$, we say that $\alpha$ is of class $\mathcal{K}_{\infty}$.
} 
0 for all $t \geq 0$ ), see, for instance, [25, Chapter 9.2]. We conjecture it is possible however to relax Assumptions 1 and 2 by requiring only locally Lipschitz functions and by restricting the attention to a semi-global framework in which solutions starts from a known compact set, see, for instance, [25, Chapter 9.3] or [24]. Since, in this work, we deal with infinite-dimensional systems, we restrict the analysis to the global framework, while keeping in mind the fact that all the analysis could be done also under milder conditions, although with possible mathematical developments that would unnecessarily over-complicate the exposition of the developments of this work.

Depending on the considered framework, system (5) may represent a scenario in which the output $e$ represents the error between a desired output and a given periodic reference (i.e., output tracking), or a scenario in which the function $q$ represents the composition of the coupling of the $(z, e)$-dynamics with a periodic perturbation affecting the $e$-dynamics (i.e., disturbance rejection). Hence, without loss of generality, the objective of this work is to design a dynamical control feedback for system (5) such that the trajectories of the overall closed-loop system are bounded (forward in time) and moreover the state-variable $e$ is regulated asymptotically to zero with a global domain of attraction, namely

$$
\lim _{t \rightarrow \infty} e(t)=0, \quad \forall(z(0), e(0)) \in \mathbb{R}^{n} \times \mathbb{R},
$$

possibly in a robust manner with respect to $f$ and $q$, since we desire to use as little information as possible about $f, q$ in the control feedback design. For instance, we would like to use only the knowledge of the constants $\alpha, \gamma, \ell_{q}$ and the period $T$ in Assumptions 1 and 2. Note that a simple high-gain feedback $u=-\kappa e$, with $\kappa>0$, cannot in general ensure (11) since the origin of the zero-dynamics is not an equilibrium and the function $q(t, \bar{z}, 0)$ is in general not equal to zero.

\section{Forwarding-Based RC-Design for Unitary Rel- ative Degree Systems}

Consider system (5). Assumption 1 states that all functions are $T$-periodic and so is the attractive steady-state $\bar{z}$ of the zero-dynamics of system (5) established by Assumption 2. A direct consequence is that in order to guarantee invariance of the solution $(z(t), e(t))=(\bar{z}(t), 0)$ for the total dynamics of (5), the control law needs to be able to generate the signal $u(t)=-q(t, \bar{z}(t), 0)$ which is $T$-periodic. Such observation justifies therefore the development of a RC-scheme in order to achieve the control objective (11) for system (5). To this end, we first equip system (5) with the internal model (1) and, as a second step, we design a new partial state-feedback law, which is a function of $e$ and $\eta(\cdot, x)$, to stabilize the extended system (1), (5). Inspired by the forwarding-stabilization technique developed in [27], we propose the following RCforwarding-based scheme, consisting of the internal model unit

$$
\begin{aligned}
\eta_{t}(t, x) & =-\frac{1}{T} \eta_{x}(t, x) & & \forall(t, x) \in \mathbb{R}_{+} \times[0,1], \\
\eta(t, 0) & =\eta(t, 1)+e(t) & & \forall t \in \mathbb{R}_{+}, \\
\eta(0, x) & =\eta_{0}(x) & & \forall x \in[0,1],
\end{aligned}
$$

with initial conditions $\eta_{0} \in L^{2}(0,1)$, and the stabilizing feedback law

$$
\begin{aligned}
& u(t)=-\kappa e(t)+v(t), \\
& v(t)=\mu \int_{0}^{1}(\eta(t, x)-M(x) e(t)) M(x) d x,
\end{aligned}
$$

where $\kappa, \mu>0$ are design parameters and the function $M:[0,1] \rightarrow \mathbb{R}$ in $(13 \mathrm{~b})$ is defined as the solution of the following two-point boundary value problem:

$$
M^{\prime}(x)=\kappa T M(x), \quad M(0)=M(1)+1 .
$$

Note that the solution to (14) is given by

$$
M(x)=\frac{\exp (\kappa T x)}{1-\exp (\kappa T)}, \quad x \in[0,1] .
$$

The main motivations justifying the RC-design (12)-(14) can be summarized as follows:

- The regulator (12) with input $e=0$ and output $v$ given by (13b) can be seen as a "universal generator" of $C^{2} T$-periodic signals, hence it satisfies the internal model property, see forthcoming Lemma 1. In particular, the objective of the internal model unit is therefore to (automatically) generate the correct steady-state input $u(t) \equiv-q(t, \bar{z}(t), 0)$, with $\bar{z}$ given by Assumption 2.

- The feedback law (13) is composed of two terms. The first term in (13a) is $-\kappa e$, which is a proportional (high-gain) feedback term needed to stabilize the $(z, e)$-dynamics, which may be unstable in openloop due to the term $q$. The second term in (13a), $v$, as defined in (13b), is needed to stabilize the $\eta$ dynamics and is based on forwarding techniques (see [23] or [27]). In particular, suppose to ignore the term $q$ (which is dominated by $\kappa e$ ) in the $e$-dynamics and consider the system $\dot{e}=-\kappa e$. Then, we can consider the $e$-dynamics to be fast and $\eta$-dynamics as slow relative to that fast time scale. As consequence, the main strategy consists in designing a feedback law, that stabilizes the $\eta$-dynamics on an invariant manifold that depends on $e$, which is designed to be linear (since we ignored the nonlinear term $q$ ) according to $M(x) e(t)$, with $M$ defined in (14). Indeed, the stabilizing action $v$ contains a term in $\eta-M e$ for this purpose. If this term converges to zero, then $v$ converges to zero and, since $e$ converges to zero when $v=0$, one can hope that both $\eta$ and $z$ converges to zero if $M$ is defined in an appropriate way. 
Following previous design ideas, we now prove the internal model property of the regulator (12), (13b).

Lemma 1. Let $M$ be the solution to (14). For any $C^{2} T$ periodic function $\psi: \mathbb{R}_{+} \rightarrow \mathbb{R}$ and any $\mu>0$, there exists $\bar{\eta}_{0} \in L^{2}(0,1)$, such that the solution to the system

$$
\begin{aligned}
\bar{\eta}_{t}(t, x) & =-\frac{1}{T} \bar{\eta}_{x}(t, x), & & \forall(t, x) \in \mathbb{R}_{+} \times[0,1], \\
\bar{\eta}(t, 0) & =\bar{\eta}(t, 1) & & \forall t \in \mathbb{R}_{+}, \\
\bar{\eta}(0, x) & =\bar{\eta}_{0}(x) & & \forall x \in[0,1], \\
\bar{v}(t) & =\mu \int_{0}^{1} \bar{\eta}(t, x) M(x) d x & & \forall t \in \mathbb{R}_{+},
\end{aligned}
$$

satisfies $\bar{v}(t) \equiv \psi(t)$ for all $t \in \mathbb{R}_{+}$.

Proof. First of all, note that, due to the boundary condition $\bar{\eta}(t, 0)=\bar{\eta}(t, 1)$, it follows from (3) that the solution to (16) is periodic, namely $\bar{\eta}(t+T, x)=\bar{\eta}(t, x)$ for all $(t, x) \in \mathbb{R}_{+} \times[0,1]$. Now, let us define the function $\Psi: \mathbb{R}_{+} \rightarrow \mathbb{R}$ as $\Psi(t):=\dot{\psi}(t)-\kappa \psi(t)$. The function $\Psi$ is $C^{1}$ and $T$-periodic due to the properties of $\psi$. Now let $\bar{\eta}_{0}(x)=\frac{T}{\mu} \Psi((1-x) T)$ for all $x \in[0,1]$. Periodicity of $\Psi$ implies $\bar{\eta}_{0}(0)=\bar{\eta}_{0}(1)$ and $C^{1}$ of $\Psi$ implies in particular $\bar{\eta}_{0} \in H^{1}(0,1)$. Furthermore, by using (3) and the above initial condition, the solution to $\bar{\eta}(t, x)$ to $(16)$ satisfies

$$
\bar{\eta}(t, x)=\bar{\eta}\left(0, x-\frac{t}{T}\right)=\frac{T}{\mu} \Psi(t+(1-x) T),
$$

for all $x \in[0,1]$ and all $0 \leq t \leq T x$. Periodicity of $\bar{\eta}$ allows to extend the previous relation to all times, namely,

$$
\bar{\eta}(t, x)=\frac{T}{\mu} \Psi(t+(1-x) T) \quad \forall(t, x) \in \mathbb{R}_{+} \times[0,1] .
$$

Therefore, by using previous relations, the definition of $M$ in (15), the expression for $\bar{v}$ as in (16), and periodicity of $\psi$, we obtain

$$
\begin{aligned}
\bar{v}(t) & =T \int_{0}^{1} \Psi(t+(1-x) T) \frac{\exp (\kappa T x)}{1-\exp (\kappa T)} d x \\
& =\int_{0}^{T} \Psi(t+T-s) \frac{\exp (\kappa s)}{1-\exp (\kappa T)} d s \\
& =\int_{0}^{T}[\dot{\psi}(t-s)-\kappa \psi(t-s)] \frac{\exp (\kappa s)}{1-\exp (\kappa T)} d s \\
& =\frac{-1}{1-\exp (\kappa T)}[\psi(t-s) \exp (\kappa s)]_{0}^{T}=\psi(t)
\end{aligned}
$$

for all $t \in[0, T]$, where in the last equality periodicity of $\psi$ is again used. Finally, since $\bar{\eta}$ is $H^{1}(0,1)$ and $T$-periodic, we conclude that the previous relation holds for all $t \in \mathbb{R}_{+}$, concluding the proof.

Clearly, Lemma 1 shows that the property that (12) with input $e=0$ and output $v$ given by (13b) is a "universal generator" of $C^{2} T$-periodic signals, for an appropriated choice of the initial conditions $\eta_{0}$. To the aim of regulation, however, such initial conditions $\eta_{0}$ do not need to be known, as they will be automatically "learned" over the time due to the stability properties of the overall closedloop system trajectories.

We can now state the main result of this work, concerning the properties of the closed-loop system (5), (12), (13). The proof is postponed to the Appendix.

Theorem 1. Suppose Assumptions 1, 2 hold. Then, there exists $\kappa^{\star} \geq 1$ (which depends on the constants $\ell_{q}, \alpha, \gamma$ defined in Assumptions 1, 2) such that, for any $\kappa \geq \kappa^{\star}$ and any $\mu>0$, the following statements hold.

(i) For any initial condition $\left(z_{0}, e_{0}, \eta_{0}\right) \in \mathbb{R}^{n} \times \mathbb{R} \times$ $L^{2}(0,1)$, the closed-loop system (5), (12), (13), (14) admits a unique solution $(z, e, \eta) \in C^{0}\left([0, \infty) ; \mathbb{R}^{n} \times\right.$ $\left.\mathbb{R} \times L^{2}(0,1)\right)$, satisfying $(z(0), e(0), \eta(0, x))=$ $\left(z_{0}, e_{0}, \eta_{0}\right)$, which is bounded for all $t \geq 0$, namely

$$
|z(t)|+|e(t)|+\|\eta(t, \cdot)\|_{L^{2}} \leq \delta, \quad \forall t \in \mathbb{R}_{+}
$$

for some $\delta>0$.

(ii) Let the set $\mathcal{A}$ be defined, for all $t \in \mathbb{R}_{+}$, as

$$
\begin{aligned}
\mathcal{A}(t):=\left\{(z, e, \eta) \in \mathbb{R}^{n} \times \mathbb{R} \times L^{2}(0,1):\right. & \\
|z(t)-\bar{z}(t)| & =0,|e(t)|=0, \\
& \left.\|\eta(t)-\bar{\eta}(t)\|_{L^{2}}=0\right\},
\end{aligned}
$$

where $\bar{z}$ is defined in Assumption 2 and $\bar{\eta}$ is the solution of system (16) for the particular choice $\psi(t)=$ $-q(t, \bar{z}(t), 0)$. Then, the set $\mathcal{A}$ is globally asymptotically stable ${ }^{5}$ for any solution of the closed-loop system (5), (12), (13), (14) starting from $\mathbb{R}^{n} \times \mathbb{R} \times L^{2}(0,1)$.

In summary, Theorem 1 states that the regulator (12)(14) solves the repetitive control problem for system (5) defined in Section 2, namely the solutions of the overall closed-loop system are bounded (forward in time) and (11) holds.

Remark. (Robustness properties) We care to stress that the regulation objective (11) is obtained robustly with respect to model uncertainties on the functions $f, q$, in the sense that we do not know the explicit knowledge of them. Indeed, for the design of the feedback law (12), (13), we only need to know the period $T$ and the constants $\alpha, \gamma, \ell_{q}$ of Assumptions 1, 2 (that will determine the value of $\kappa^{\star}$ of the statement of Theorem 1, see (A.8) in the proof), and not the functions $f, q$ itself. As a consequence, the result of Theorem 1 holds robustly for all such functions satisfying these assumptions.

Remark. (Alternative Time-Domain Representation) In view of Theorem 1, we can select, without loss

\footnotetext{
${ }^{5}$ See, for instance, $[30$, Chapter $4, \S 15]$ for the definition of set stability. Note that, because of the boundary condition $\eta(t, 0)=\eta(t, 1)$ of the transport equation, exponential stability of the closed-loop system cannot be guaranteed in view of the presence of an infinitenumber of poles on the imaginary axis and the use of a bounded (in the sense of [31, Page 24]) control operator. See also [20, 21] for other examples of such phenomenon.
} 
of generality, the initial condition $\eta(0, x)=0$ for the regulator (12). With such a choice, the solution to (12) is given by $\eta(t, 0)=e(t)+e(t-T)$ for all $t \geq T$. Hence, by using the explicit solution (3) of a transport equation, it is readily seen that $\eta(t, x)=e(t-x T, 0)+e(t-x T-T)$. As a consequence, by operating a change of coordinates $x \mapsto s:=T x$, and using the definition of $M$ in (15), the resulting repetitive-control based regulator (12)-(13) can be alternatively written, in the pure time-domain (i.e., without the use of the transport-equation), as

$$
\begin{aligned}
& u(t)=-\left(\kappa+\frac{\mu}{2 \kappa T} \frac{\exp (2 \kappa T)-1}{(\exp (\kappa T)-1)^{2}}\right) e(t)+v(t) \\
& v(t)=\frac{\mu}{T} \int_{0}^{T} \frac{\exp (\kappa s)}{1-\exp (\kappa T)}[e(t-s)+e(t-s-T)] d s
\end{aligned}
$$

where $e(t) \equiv 0$ for all $t<0$. The transport-equation representation (2) is therefore not needed for the feedbackimplementation, although it is instrumental for obtaining the above feedback law and essential to establish the stability results of Theorem 1 .

\section{RC-Design for Higher Relative Degree Systems via Partial State-Feedback}

In this section, we present a RC-approach for systems with relative degree higher than one, that is, for minimumphase systems of arbitrary relative degree $r$ of the form

$$
\begin{aligned}
\dot{z} & =f\left(t, z, \xi_{1}\right), \\
\dot{\xi}_{i} & =\xi_{i+1}, \quad i=1, \ldots, r-1 \\
\dot{\xi}_{r} & =q\left(t, z, \xi_{1}, \ldots, \xi_{r}\right)+u, \\
e & =\xi_{1},
\end{aligned}
$$

where $\left(z^{\top}, \xi^{\top}\right)^{\top} \in \mathbb{R}^{n} \times \mathbb{R}^{r}$ is the system state, with the $z \in \mathbb{R}^{n}$-dynamics being the zero-dynamics and $\xi:=$ $\left(\xi_{1}, \ldots, \xi_{r}\right) \in \mathbb{R}^{r}$ representing the derivatives of the output $e$ that we aim to regulate to zero. We suppose that the functions $f, q$ in (19) satisfy the following assumption.

Assumption 3. The functions $f, q$ in (19) are globally Lipschitz, $C^{2}$ in their arguments and periodic with respect to the first argument. Moreover, the function $f$ satisfies 6 Assumption 2.

Based on the design proposed in Section 3, in order to solve the control objective (11) for system (19), we propose the following regulator:

$$
\begin{aligned}
\eta_{t}(t, x) & =-\frac{1}{T} \eta_{x}(t, x) \\
\eta(t, 0) & =\eta(t, 1)+\theta(t) \\
\eta(0, x) & =\eta_{0}(x) \\
u(t) & =-\kappa \theta(t)+\mu \int_{0}^{1}(\eta(t, x)-M(x) \theta(t)) M(x) d x
\end{aligned}
$$

\footnotetext{
${ }^{6}$ where in inequality (10), the variable $e$ has to be replaced by $\xi_{1}$.
}

with $M$ defined as in (14) and $\theta$ as

$$
\theta:=\xi_{r}+\sum_{i=1}^{r-1} g^{r-i} a_{i} \xi_{i}
$$

with $g>0$ and $a_{i}>0, i=1, \ldots, r-1$, parameters to be defined. We now can present the following result on the properties of the resulting closed-loop system (19), (20).

Theorem 2. Suppose Assumption 3 holds. Let $a_{i}>0$, $i=1, \ldots, r-1$, be selected such that the polynomial $p_{0}(\lambda):=\lambda^{r-1}+a_{r-1} \lambda^{r-2}+\cdots+a_{2} \lambda+a_{1}$ is Hurwitz. Then, there exists $g^{\star} \geq 1$ and, for any $g>g^{\star}$, there exists $\kappa^{\star} \geq 1$ (which depends on the constants $\ell_{q}, \alpha, \gamma$ defined in Assumptions 1, 2, and the choice of $g$ and $a_{1}, \ldots, a_{r-1}$ ) such that, for any $\kappa \geq \kappa^{\star}$ and any $\mu>0$, the following statements hold.

(i) For any initial condition $\left(z_{0}, \xi_{0}, \eta_{0}\right) \in \mathbb{R}^{n} \times \mathbb{R}^{r} \times$ $L^{2}(0,1)$, the closed-loop system (19), (20), (21), admits a unique solution $(z, \xi, \eta) \in C^{0}\left([0, \infty) ; \mathbb{R}^{n} \times \mathbb{R}^{r} \times\right.$ $\left.L^{2}(0,1)\right)$, satisfying $(z(0), \xi(0), \eta(0, x))=\left(z_{0}, \xi_{0}, \eta_{0}\right)$, which is bounded for all $t \geq 0$, namely

$$
|z(t)|+|\xi(t)|+\|\eta(t, \cdot)\|_{L^{2}} \leq \delta, \quad \forall t \in \mathbb{R}_{+}
$$

for some $\delta>0$.

(ii) Let the set $\mathcal{A}$ be defined, for all $t \in \mathbb{R}_{+}$, as

$$
\begin{aligned}
& \mathcal{A}(t):=\left\{(z, \xi, \eta) \in \mathbb{R}^{n} \times \mathbb{R}^{r} \times L^{2}(0,1):\right. \\
& \left.|z(t)-\bar{z}(t)|=0,|\xi(t)|=0,\|\eta(t)-\bar{\eta}(t)\|_{L^{2}}=0\right\},
\end{aligned}
$$

where $\bar{z}$ is defined in Assumption 2 and $\bar{\eta}$ is the solution of system (16) for the particular choice $\psi(t)=$ $-q(t, \bar{z}(t), 0)$. Then, the set $\mathcal{A}$ is globally asymptotically stable for any solution of the closed-loop system (19), (20), (21), starting from $\mathbb{R}^{n} \times \mathbb{R}^{r} \times L^{2}(0,1)$.

Proof. Following [24, Section V-A], the main idea of the proof consists in transforming system (19) into a system of unitary relative degree by means of a linear change of coordinates, and then to re-apply the results of Theorem 1. In particular, with the definition of $\theta$ given in (21) in mind, by applying the following change of coordinates

$$
\begin{aligned}
\xi_{i} & \mapsto \varepsilon_{i}:=g^{r-i} \xi_{i}, & i=1, \ldots, r-1, \\
\xi_{r} & \mapsto \theta:=\xi_{r}+\sum_{i=1}^{r-1} g^{r-i} a_{i} \xi_{i}, &
\end{aligned}
$$

to system (19), we obtain the transformed dynamics

$$
\begin{aligned}
\dot{z} & =f\left(t, z, g^{1-r} C \varepsilon\right) \\
\dot{\varepsilon} & =g A \varepsilon+g B \theta \\
\dot{\theta} & =q_{0}(t, \varepsilon, \theta)+q_{1}(\varepsilon, \theta)+u \\
e & =g^{1-r} C \varepsilon,
\end{aligned}
$$

in which $\varepsilon:=\left(\varepsilon_{1}, \ldots, \varepsilon_{r-1}\right)^{\top} \in \mathbb{R}^{r-1}, B:=\left(\begin{array}{ll}0_{r-2,1} & 1\end{array}\right)^{\top}$,

$$
\begin{gathered}
C:=\left(\begin{array}{cc}
1 & 0_{1 \times r-2}
\end{array}\right), A:=\left(\begin{array}{cc}
0_{r-2,1} & I_{r-2} \\
-a_{1} & -a_{2} \cdots-a_{r-1}
\end{array}\right), \\
q_{0}(t, \varepsilon, \theta):=q\left(t, z, g^{1-r} \varepsilon_{1}, \ldots, g^{-1} \varepsilon_{r-1}, \theta-\sum_{i=1}^{r-1} a_{i} \varepsilon_{i}\right), \\
q_{1}(\varepsilon, \theta):=g \sum_{i=1}^{r-2} a_{i} \varepsilon_{i+1}+g a_{r-1}\left(\theta-\sum_{i=1}^{r-1} a_{i} \varepsilon_{i}\right) .
\end{gathered}
$$


Now recall that the subsystem $z$ is input-to-state stable with respect to $\xi_{1}$ and therefore to $g^{1-r} C \varepsilon$. Indeed, under Assumption 3 (and therefore by Assumption 2), inequality (10), in which $e$ is substituted with $\xi_{1}$, holds. Furthermore, the matrix $A$ is Hurwitz by construction since it is the companion form of the Hurwitz polynomial $p(\lambda)$. As a consequence, for $g$ large enough, system (22) with output $\theta$ and zero-dynamics $(z, \varepsilon)$ is still minimum-phase. In other words, the subsystem $(z, \varepsilon)$ is input-to-state stable with respect to $\theta$. This, in particular, can be established by means of the Lyapunov function

$$
W(t, z, \xi)=V(t, z)+\varepsilon^{\top} P \varepsilon
$$

with $V$ satisfying (9), (10), and $P$ solution to the Lyapunov equation $P A+A^{\top} P=-2 I$. Indeed, by using (10), we can compute the time-derivative of $W$ along the solutions of the closed-loop system $(22),(20)$, denoted as $\dot{W}(t)$, as

$$
\begin{aligned}
\dot{W}(t) & \leq-\alpha|z-\bar{z}(t)|^{2}+\gamma g^{1-r}|C \varepsilon|-2 g|\varepsilon|^{2}+2 g \varepsilon^{\top} P B \theta \\
& \leq-\alpha|z-\bar{z}(t)|^{2}-(g-\gamma)|\varepsilon|^{2}+g|P|^{2} \theta^{2}
\end{aligned}
$$

for all $t \in \mathbb{R}_{+}$, showing the desired input-to-state stability properties for $g>g^{\star}, g^{\star}=\max \{1, \gamma\}$. Also, we deduce that the equilibrium of the zero-dynamics of (22) is given by $(z, \varepsilon)=(\bar{z}, 0)$. Furthermore, it follows by linearity of the coordinate transformation that if $q$ is a $C^{2} T$-periodic globally Lipschitz function, then so are the functions $q_{0}, q_{1}$ defined in (22).

We conclude therefore that the system (22) satisfies the assumptions of Theorem 1 when considered as a unitaryrelative degree system with $(z, \varepsilon)$ zero-dynamics, that is, a system of the form (5). We can therefore apply Theorem 1 to show that solutions of the closed-loop system (22), (20) are bounded (forward in time) and converges asymptotically to the set

$$
\begin{gathered}
\left\{(z, \varepsilon, \theta, \eta) \in \mathbb{R}^{n} \times \mathbb{R}^{r-1} \times \mathbb{R} \times L^{2}(0,1):|z-\bar{z}(t)|=0,\right. \\
\left.|\varepsilon(t)|=0,|\theta(t)|=0,\|\eta(t)-\bar{\eta}(t)\|_{L^{2}}=0\right\}
\end{gathered}
$$

for $\kappa$ large enough and any $\mu>0$. Note that the value of $\kappa^{\star}$ depends on the choice of $g$ which has to be selected first. By construction, the set $\left\{(\varepsilon, \theta) \in \mathbb{R}^{r-1} \times \mathbb{R}: \varepsilon=0, \theta=0\right\}$, corresponds to the set $\{\xi=0\}$, concluding the proof.

\section{Some Illustrative Examples}

The literature on control problem for minimum-phase systems is vast and and hence so is the number of potential applications to which the results in this paper could be applied. In this section, we concisely discuss three applications of the proposed RC-design technique developed in Sections 3 and 4.

\subsection{Tracking control for frictional systems with unknown friction characteristics.}

Friction is an ubiquitous phenomenon in mechanical systems that is difficult to model and often requires to be compensated, for instance, in order to achieve required positioning performance in high-tech motion systems. Consider a two-dimensional (normalized) mechanical system of the form

$$
\ddot{\vartheta}=F(\dot{\vartheta})+u
$$

where position $\vartheta$ and speed $\dot{\vartheta}$ are measurable, $u \in \mathbb{R}$ is the control force and $F$ is an unknown (smooth enough) globally Lipschitz function characterizing the friction. Suppose that we desire the controller to ensure $\vartheta$ to asymptotically track any arbitrary periodic reference $\vartheta_{\text {ref }}(t)$ with known bounded first and second-order derivatives. By selecting the error coordinates

$$
\xi_{1}:=\vartheta-\vartheta_{\text {ref }}(t), \quad \xi_{2}:=\dot{\vartheta}-\dot{\vartheta}_{\text {ref }}(t),
$$

we obtain a system in the form (19), with $n=2$, in which there is no zero-dynamics $z$ and the function $q$ is given by

$$
q(t, \xi):=F\left(\xi_{2}+\dot{\vartheta}_{\text {ref }}(t)\right)-\ddot{\vartheta}_{\text {ref }}(t),
$$

which is $T$-periodic and globally Lipschitz. As the system has no-zero dynamics, the minimum-phaseness prerequisite is trivially verified. Assumption 3 is therefore satisfied. Hence, we can employ the RC-design of Section 4 to address the desired tracking problem. Note that for the design of the the regulator (20), only the knowledge of a (possible conservative) approximation of the Lipschitz constant of the friction characteristic $F$ is required, which is beneficial given the typical uncertainty in friction models. As a consequence Theorem 2 can be used to show that a controller of the form (12), (13), (14) solves the periodic tracking problem at hand.

\subsection{Neural Network Function Approximation}

As discussed in [32], many papers in neural network control have been devoted to the solution of the tracking problem for the $n$-dimensional system

$$
\begin{aligned}
& \dot{\chi}_{i}=\chi_{i+1}, \quad i=1, \ldots, n-1, \\
& \dot{\chi}_{n}=\sum_{i=1}^{n} a_{i} \chi_{i}+\sum_{i=1}^{N} \frac{\delta_{i}}{1+\alpha_{i} \exp \left(-\beta_{i} \chi_{i}\right)}+u
\end{aligned}
$$

where $N$ is a known positive integer and all the parameters $a_{i}, i=1, \ldots, n$, and $\alpha_{i}, \beta_{i}, \delta_{i}>0, i=1, \ldots, N$, are unknown. In particular, we can apply the design procedure of Section 4 to design a state-feedback law ensuring $\chi_{1}$ to asymptotically track a desired periodic smooth reference $\chi_{\text {ref }}(t)$. By setting

$$
\xi_{i}:=\chi_{1}-\chi_{\mathrm{ref}}^{(i-1)}(t), \quad i=1, \ldots, n,
$$

the system is transformed into the form (19) in which there is no zero-dynamics and the function $q$ is given by

$$
\begin{aligned}
q(t, \xi):= & -\chi_{\mathrm{ref}}^{(n)}(t)+\sum_{i=1}^{n} a_{i}\left(\xi_{i}+\chi_{\mathrm{ref}}^{(i-1)}(t)\right) \\
& +\sum_{i=1}^{N} \frac{\delta_{i}}{1+\alpha_{i} \exp \left(-\beta_{i}\left(\xi_{i}+\chi_{\mathrm{ref}}^{(i-1)}(t)\right)\right.},
\end{aligned}
$$




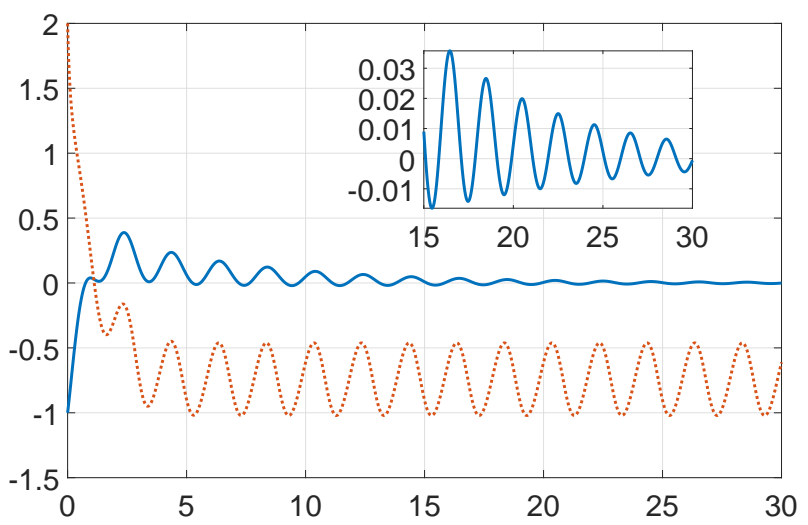

Figure 2: Closed-loop system (23), (12), (13): trajectories of $z(t)$ (dotted red line) and $e(t)$ (blue line) for $t \in[0,30]$.

which is $T$-periodic and globally Lipschitz. In order to design the control law (20), we need to suppose that the parameters $\delta_{i}, a_{i}$, satisfy $\sum_{i=1}^{n}\left|a_{i}\right|+\sum_{i=1}^{N}\left|\delta_{i} \beta_{i}\right| \leq \bar{c}$ for some known $\bar{c}>0$. Indeed, in this case, an overapproximation of the Lipschitz constant of the function $q$ is given by $\ell_{q}=\bar{c}$. As the system has no-zero dynamics, the minimum-phaseness prerequisite is trivially verified. Hence Assumption 3 is satisfied and we can use apply Theorem 2 to show that a controller of the form $(12),(13),(14)$ solves the periodic tracking problem at hand.

\subsection{Simulation Example}

As an illustrative simulation example, consider the following system

$$
\begin{aligned}
\dot{z} & =-z^{3}+\cos (2 \pi t)-0.5+e \\
\dot{e} & =0.5+2 \arctan (z) e+u
\end{aligned}
$$

verifying Assumptions 1 and 2 with $T=1$ on any given compact set, and select the controller (12), (13), (14) with $\kappa=2$ and $\mu=10$. Figure 2 (resp., Figure 3 ) shows the evolution of the trajectories of $(z, e)$ (resp., $\|\eta(t, \cdot)\|_{L^{2}}$ ), for the initial conditions $\left(z_{0}, e_{0}\right)=(1,-1)$ and $\eta_{0}: x \rightarrow \sin (x)$. Simulations have been performed using the method of characteristics (see, e.g., [33, Section 2.1]) to compute the exact solution of (12) at any time with a spatial discretization interval of $10^{-2}$, while the ODEs have been discretized using the Euler method with time step $10^{-2}$. Figure 2 shows the asymptotic convergence of $e$ to zero with a slow residual dynamics typical of forwarding approaches.

\section{Conclusions}

In this work, we addressed the problem of repetitive control for nonlinear systems. Differently from standard approaches, we represent here the delay as a transport equation and we propose a new forwarding-based (partial) state feedback that uses not only the boundary information of

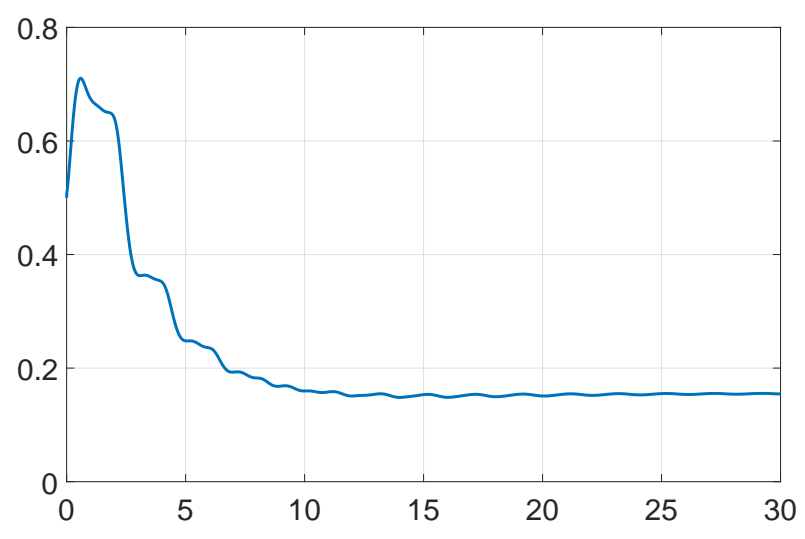

Figure 3: Closed-loop system (23), (12), (13): evolution of $\|\eta(t, \cdot)\|_{L^{2}}$ for $t \in[0,30]$.

the transport equation representing the delay, but all its state. This allows to establish asymptotic stability of a RC-scheme for nonlinear minimum-phase systems with arbitrarily relative degree and constant high-frequency gain.

Implementation issues related to the (time and/or space) discretization of the regulator (20) have not been discussed in this manuscript, since providing a numerical scheme for spatio-temporal discretization would need a careful study of its convergence, and this is out of the (theoretical) scope of this brief article. Nevertheless, a proper discretization of the proposed infinite-dimensional scheme surely deserves of further research, since different approaches can be employed, such as the use of low-pass filters $[1,17,18]$, Fourier approximations [19] [22], [20] or discrete-delay representations $[3,6,10]$. It is worth stressing, that the proposed approach also opens the doors for new results in RC-design for discrete-time nonlinear systems, possibly combining recent results in forwarding stabilization [34].

From the theoretical point of view, future works include the extension of the proposed technique to pure output-feedback designs for higher relative degree systems; the study of RC-schemes for non-minimum phase systems and/or multi-variable systems; the use of adaptive schemes that possibly estimate online the period $T$; and the extension of the proposed technique to the context of cooperative output control problems for multi-agent systems, see, e.g., $[35,36]$.

\section{Appendix A. Proof of Theorem 1}

The proof of Theorem 1 is organized as follows. First, we modify system (5), (12), (13) by means of some changes of coordinates. Existence and uniqueness of solutions is discussed first. Finally, a Lyapunov-based analysis is presented, allowing to establish boundedness of solutions and convergence to the desired stead-state. 
Let $\psi(t):=-q(t, \bar{z}(t), 0)$, with $\bar{z}$ given by Assumption 2 . In view of Assumptions 1 and 2 , the function $\psi$ is $C^{2}$ and $T$-periodic. Hence, consider the following change of coordinates

$$
\left(\begin{array}{c}
z(t) \\
e(t) \\
\eta(t)
\end{array}\right) \mapsto\left(\begin{array}{c}
\zeta(t) \\
e(t) \\
\phi(t)
\end{array}\right):=\left(\begin{array}{c}
z-\bar{z}(t) \\
e(t) \\
\eta(t, x)-\bar{\eta}(t, x)-M(x) e(t)
\end{array}\right)
$$

where $\bar{\eta}$ is the solution of (16) satisfying $\bar{v}(t) \equiv \psi(t)=$ $-q(t, \bar{z}(t), 0)$ for all $t \geq 0$, and $\bar{z}$ is defined by Assumption 2. By using the following identity

$$
-\frac{1}{T}\left[\eta_{x}(t, x)-\bar{\eta}_{x}(t, x)\right]+\kappa M(x) e(t)=-\frac{1}{T} \phi_{x}(t, x)
$$

obtained from the definition of $M$ in (14), we obtain

$$
\begin{aligned}
\dot{\zeta}(t) & =F(t, \zeta, e) \\
\dot{e}(t) & =\Delta(t, \zeta, e)-\kappa e(t)+\mu \int_{0}^{1} \phi(t, x) M(x) d x \\
\phi_{t}(t, x) & =-\frac{1}{T} \phi_{x}(t, x)-M(x) \Delta(t, \zeta, e) \\
\phi(t, 0) & =\phi(t, 1) \quad-\mu M(x) \int_{0}^{1} \phi(t, x) M(x) d x \\
\phi(0, x) & =\phi_{0}(x)
\end{aligned}
$$

where $\phi_{0}(x):=\eta_{0}(x)-\bar{\eta}_{0}(x)-M(x) e(0) \in H^{1}(0,1)$, and the functions $F, \Delta$, defined as

$$
\begin{aligned}
F(t, \zeta, e) & :=f(t, \zeta+\bar{z}(t), e)-f(t, \bar{z}(t), 0), \\
\Delta(t, \zeta, e) & :=q(t, \zeta+\bar{z}(t), e)-q(t, \bar{z}(t), 0),
\end{aligned}
$$

satisfy, in view of Assumption 1, the following inequalities:

$$
\begin{aligned}
& |F(t, \zeta, e)| \leq \ell_{f}|\zeta|+\ell_{f}|e|, \\
& |\Delta(t, \zeta, e)| \leq \ell_{q}|\zeta|+\ell_{q}|e|,
\end{aligned}
$$

for all $(t, \zeta, e) \in \mathbb{R}_{+} \times \mathbb{R}^{n} \times \mathbb{R}$. Note that, by construction, $F(t, 0,0)=0$ and $\Delta(t, 0,0)=0$ for all $t \in \mathbb{R}_{+}$. Hence, it can be verified that the origin $(\zeta, e, \phi)=(0,0,0)$ in the $\mathbb{R}^{n} \times \mathbb{R} \times L^{2}(0,1)$-topology is an equilibrium point of (A.1). Furthermore, by recalling the definitions of $\zeta$ and $\phi$, we have the existence of class $\mathcal{K}_{\infty}$ functions $\beta$ and $\bar{\beta}$ satisfying

$$
\begin{aligned}
& \beta\left(|z-\bar{z}(t)|+|e|+\|\eta-\bar{\eta}\|_{L^{2}}\right) \\
& \leq|\zeta|+|e|+\|\phi\|_{L^{2}} \leq \beta\left(|z-\bar{z}(t)|+|e|+\|\eta-\bar{\eta}\|_{L^{2}}\right) .
\end{aligned}
$$

Therefore, showing that the origin $(\zeta, e, \phi(\cdot, x))=0$ of (A.1) is stable, coincides, in the original coordinates $(z, e, \eta)$, in showing that the set $\mathcal{A}$ defined in (18) is stable. In other words, the items (i) and (ii) of Theorem 1 will be equivalently proved in the coordinates (A.1).

Using the Duhamel formula (i.e., variation of constants formula), one can write the solution to (A.1) with an integral formula which exists thanks to a Banach fixed point theorem, as it has been done in [37]. Thus, one can prove that solutions of the system (A.1) exist and are unique for some small interval of time, uniformly in the initial time $t_{0}$ and in the initial conditions $\left(\zeta_{0}, e_{0}, \phi_{0}\right)$. This is stated in the next lemma. We omit the proof for space reasons.
Lemma 2 (Well-posedness of (A.1)). For any $t_{0}>0$ and any initial conditions $\left(\zeta_{0}, e_{0}, \phi_{0}\right) \in \mathbb{R}^{n} \times \mathbb{R} \times L^{2}(0,1)$ (resp. $\mathbb{R}^{n} \times \mathbb{R} \times H^{1}(0,1)$ with the compatibility condition $\left.\phi_{0}(0)=\phi_{0}(1)\right)$, there exists a positive constant $\tau>0$ sufficiently small such that there exists a unique solution $(\zeta, e, \phi) \in C^{0}\left(\left[t_{0}, t_{0}+\tau\right] ; \mathbb{R}^{n} \times \mathbb{R} \times L^{2}(0,1)\right.$ ) (resp. $C^{1}\left(\left[t_{0}, t_{0}+\tau\right] ; \mathbb{R}^{n} \times \mathbb{R} \times L^{2}(0,1)\right) \cap C^{0}\left(\left[t_{0}, t_{0}+\tau\right] ; \mathbb{R}^{n} \times \mathbb{R} \times\right.$ $\left.\left.H^{1}(0,1)\right)\right)$ to system (A.1).

Now consider the Lyapunov functional

$$
U(t, \zeta, e, \phi):=c V(t, \zeta)+e^{2}+\mu \int_{0}^{1} \phi(t, x)^{2} d x,
$$

with $c>0$ to be selected and $V$ given by Assumption 2 . In view of Assumption 2, the function $U$ satisfies, for all $(t, \zeta, e, \phi) \in \mathbb{R}_{+} \times \mathbb{R}^{n} \times \mathbb{R} \times L^{2}(0,1)$,

$$
\begin{aligned}
& \underline{\alpha}_{U}\left(|\zeta|+|e|+|\phi|_{L^{2}(0,1)}\right) \leq \\
& \quad U(t, \zeta, e, w) \leq \bar{\alpha}_{U}\left(|\zeta|+|e|+|\phi|_{L^{2}(0,1)}\right),
\end{aligned}
$$

for some class $\mathcal{K}_{\infty}$ functions $\underline{\alpha}_{U}, \bar{\alpha}_{U}$. Define the set

$$
\begin{array}{r}
\Omega_{\bar{U}}:=\left\{(t, \zeta, e, \phi) \in \mathbb{R}_{+} \times \mathbb{R}^{n} \times \mathbb{R} \times L^{2}(0,1):\right. \\
U(t, \zeta, e, \phi) \leq \bar{U}\},
\end{array}
$$

for some given $\bar{U}>0$. By definition the set $\Omega_{\bar{U}}$ is a bounded subset of $\mathbb{R}^{n} \times \mathbb{R} \times L^{2}(0,1)$. Hence, consider any initial condition $\left(\zeta_{0}, e_{0}, \phi_{0}\right) \in \Omega_{\bar{U}}$. In view of Lemma 2 , there exists $\bar{\tau}>0$ such that $\left[t_{0}, t_{0}+\bar{\tau}\right)$ is the maximal interval of existence of the corresponding $\operatorname{solutions}(\zeta, e, \phi)$ to system (A.1). Note that since all the bounds and constants selected in the proof are uniform in $t_{0}$ (in particular, they do not depend on it), without loss of generality, in the reset of the proof we simply set $t_{0}=0$.

We compactly denote by $U(t)$ the expression for $U$ along such solutions. By using (10), the derivative of $U$ satisfies

$$
\begin{aligned}
\dot{U}(t) \leq & -c \alpha|\zeta|^{2}+c \gamma|e|^{2}+2 e \Delta(t, \zeta, e)-2 \kappa|e|^{2} \\
& +2 e \mu \int_{0}^{1} \phi(t, x) M(x) d x-2 \frac{\mu}{T} \int_{0}^{1} \phi(t, x) \phi_{x}(t, x) d x \\
& -2 \mu \int_{0}^{1} \phi(t, x) M(x) \Delta(t, \zeta, e) d x \\
& -2\left(\mu \int_{0}^{1} \phi(t, x) M(x) d x\right)^{2} .
\end{aligned}
$$

Note that, by using the fact that $\phi(t, 0)=\phi(t, 1)$, we obtain

$$
\int_{0}^{1} \phi(t, x) \phi_{x}(t, x) d x=0 .
$$

Then, by using Young's inequality ${ }^{7}$ and Lipschitz properties of $\Delta$ in (A.5), we have the following bounds:

$$
2 e \Delta(t, \zeta, e) \leq 2 \ell_{q}|e|(|e|+|\zeta|) \leq 3 \ell_{q}|e|^{2}+\ell_{q}|\zeta|^{2}
$$

\footnotetext{
${ }^{7}$ In particular, $2 a b \leq \nu a^{2}+\nu^{-1} b^{2}$ for any $\nu>0$.
} 
and

$$
\begin{aligned}
-2 \mu & \int_{0}^{1} \phi(t, x) M(x) \Delta(t, \zeta, e) d x \\
& \leq 2|\Delta(t, \zeta, e)|^{2}+\frac{1}{2}\left(\mu \int_{0}^{1} \phi(t, x) M(x) d x\right)^{2} \\
& \leq 4 \ell_{q}^{2}|\zeta|^{2}+4 \ell_{q}^{2}|e|^{2}+\frac{1}{2}\left(\mu \int_{0}^{1} \phi(t, x) M(x) d x\right)^{2} .
\end{aligned}
$$

Hence, combining all the bounds, we obtain

$$
\begin{aligned}
\dot{U}(t) \leq & -\left(c \alpha-4 \ell_{q}^{2}-\ell_{q}\right)|\zeta|^{2}-\left(\mu \int_{0}^{1} \phi(t, x) M(x) d x\right)^{2} \\
& -\left(2 \kappa-c \gamma-4 \ell_{q}^{2}-3 \ell_{q}-1\right)|e|^{2} .
\end{aligned}
$$

Therefore, by selecting

$$
c=\frac{4 \ell_{q}^{2}+\ell_{q}+\epsilon}{\alpha}, \quad \kappa^{\star}=\frac{1}{2}\left(c \gamma+4 \ell_{q}^{2}+3 \ell_{q}+1+\epsilon\right),
$$

for any arbitrarily small $\epsilon>0$, the previous inequality gives, for any $\kappa \geq \kappa^{\star}$,

$$
\begin{aligned}
\dot{U}(t) & \leq-\epsilon\left(|\zeta|^{2}+|e|^{2}\right)-\left(\mu \int_{0}^{1} \phi(t, x) M(x) d x\right)^{2} \\
& \leq 0 .
\end{aligned}
$$

The second inequality in (A.9) implies also $U(t) \leq U(0)$ for all $t \in[0, \bar{\tau})$. This shows that the solution $(\zeta, e, \phi)$ remain in $\Omega_{\bar{U}}$ for all (positive) times (and any $t_{0}$ ). So, from its definition, $\bar{\tau}$ is infinite, namely the solution exists for all $t \in \mathbb{R}_{+}$, and in particular satisfies

$$
|\zeta(t)|+|e(t)|+|\phi(t, \cdot)|_{L^{2}(0,1)} \leq \underline{\alpha}_{U}^{-1}(\bar{U})
$$

for all $t \geq \mathbb{R}_{+}$, where we used (A.7). Finally, the inequality (A.6) can be obtained by combining inequality (A.10) with (A.6) and the following bounds

$$
\begin{gathered}
|z|=|z-\bar{z}+\bar{z}| \leq|z-\bar{z}|+|\bar{z}| \\
\|\eta\|_{L^{2}}=\|\eta-\bar{\eta}+\bar{\eta}\|_{L^{2}} \leq\|\eta-\bar{\eta}\|_{L^{2}}+\|\bar{\eta}\|_{L^{2}}
\end{gathered}
$$

and selecting $\delta:=\underline{\beta}^{-1}\left(\underline{\alpha}_{U}^{-1}(\bar{U})\right)+\sup _{t \in[0, T]}|\bar{z}(t)|+\left\|\bar{\eta}_{0}\right\|_{L^{2}}$. Note that in view of Assumption 2 and Lemma 1, $\bar{z}$ and $\bar{\eta}_{0}$ are bounded. This concludes the proof of the item (i) of Theorem 1. Next, in order to show item (ii), we follow the main arguments used in proof of [27, Theorem 2], which are adapted to the analysis of system (A.1). In particular, by using the first inequality of (A.9), we also obtain

$$
\begin{aligned}
U(t)-U(0) \leq-\int_{0}^{t} \epsilon\left(|\zeta(s)|^{2}+|e(s)|^{2}\right) d s & \\
& -\left(\mu \int_{0}^{1} \phi(s, x) M(x) d x\right)^{2} d s,
\end{aligned}
$$

for any $t \in \mathbb{R}_{+}$. Hence, by integrating on $[0,+\infty)$, we obtain $\int_{0}^{+\infty}|\zeta(t)|^{2} d t<+\infty, \int_{0}^{+\infty}|e(t)|^{2} d t<+\infty$ and $\int_{0}^{+\infty}\left(\int_{0}^{1} \phi(t, x) M(x) d x\right)^{2} d t<+\infty$. Such inequalities, combined with the bound (A.10) and the fact that all functions are globally Lipschitz, implies that also the functions $t \mapsto e(t), t \mapsto \zeta(t)$, and $t \mapsto \int_{0}^{1} \phi(t, x) M(x) d x$ are also globally Lipschitz and therefore uniformly continuous. Hence, by applying Barbalat's lemma, we obtain $\lim _{t \rightarrow+\infty}|\zeta(t)|=0, \lim _{t \rightarrow+\infty}\left|\int_{0}^{1} \phi(t, x) M(x) d x\right|=0$ and $\lim _{t \rightarrow+\infty}|e(t)|=0$. Finally, for the last part of the proof, one can follow the same steps of the proof of [27, Theorem 2], where it can be shown, by using precompactness of solutions and LaSalle's invariance principle arguments for infinite-dimensional systems (see [38, Theorem 3.1]), that $(\zeta, e, \phi)$ converge asymptotically to $(0,0,0)$ in the $\mathbb{R} \times L^{2}(0,1)$-topology. Standard density argument (see, e.g., [39, Lemma 1]) allows also to conclude that such a result holds for initial condition $\left(\zeta_{0}, e_{0}, \phi_{0}\right)$ in $\mathbb{R} \times L^{2}(0,1)$. Therefore, the proof concludes by noting that the origin of (A.1) coincides, in the $z, e, \eta$ coordinates, with $(z, e, \eta)=(\bar{z}, 0, \bar{\eta})$, that is the set $\mathcal{A}$ defined in (18).

\section{References}

[1] S. Hara, Y. Yamamoto, T. Omata, M. Nakano, Repetitive control system: A new type servo system for periodic exogenous signals, IEEE Transactions on automatic control 33 (7) (1988) 659-668.

[2] B. A. Francis, W. M. Wonham, The internal model principle of control theory, Automatica 12 (5) (1976) 457-465.

[3] M. Tomizuka, T.-C. Tsao, K.-K. Chew, Discrete-time domain analysis and synthesis of repetitive controllers, in: IEEE American Control Conference, 1988, pp. 860-866.

[4] R. W. Longman, On the theory and design of linear repetitive control systems, European Journal of Control 16 (5) (2010) 447496.

[5] G. Escobar, P. Mattavelli, M. Hernandez-Gomez, P. R. Martinez-Rodriguez, Filters with linear-phase properties for repetitive feedback, IEEE Transactions on Industrial Electronics 61 (1) (2013) 405-413.

[6] D. Owens, M. Tomas-Rodriguez, J. Hatönen, L. Li, Discrete time linear optimal multi-periodic repetitive control: a benchmark tracking solution, International Journal of Control 79 (9) (2006) 991-1001.

[7] B. Zhang, K. Zhou, D. Wang, Multirate repetitive control for pwm dc/ac converters, IEEE Transactions on Industrial Electronics 61 (6) (2013) 2883-2890.

[8] R. W. Longman, Iterative learning control and repetitive control for engineering practice, International journal of control 73 (10) (2000) 930-954.

[9] Y. Wang, F. Gao, F. J. Doyle III, Survey on iterative learning control, repetitive control, and run-to-run control, Journal of Process Control 19 (10) (2009) 1589-1600.

[10] L. Wang, C. T. Freeman, E. Rogers, Predictive iterative learning control with experimental validation, Control Engineering Practice 53 (2016) 24-34.

[11] J. Kasac, B. Novakovic, D. Majetic, D. Brezak, Passive finitedimensional repetitive control of robot manipulators, IEEE Transactions on Control Systems Technology 16 (3) (2008) 570576.

[12] E. Kurniawan, Z. Cao, O. Mahendra, R. Wardoyo, A survey on robust repetitive control and applications, in: 2014 IEEE International Conference on Control System, Computing and Engineering, 2014, pp. 524-529.

[13] L. Blanken, S. Koekebakker, T. Oomen, Multivariable repetitive control: Decentralized designs with application to continuous media flow printing, IEEE/ASME Transactions on Mechatronics.

[14] P. Mattavelli, F. P. Marafao, Repetitive-based control for selective harmonic compensation in active power filters, IEEE Transactions on Industrial Electronics 51 (5) (2004) 1018-1024. 
[15] F. Califano, M. Bin, A. Macchelli, C. Melchiorri, Stability analysis of nonlinear repetitive control schemes, IEEE control systems letters 2 (4) (2018) 773-778.

[16] F. Califano, A. Macchelli, A stability analysis based on dissipativity of linear and nonlinear repetitive control, IFACPapersOnLine 52 (2) (2019) 40-45.

[17] T. Omata, S. Hara, M. Nakano, Nonlinear repetitive control with application to trajectory control of manipulators, Journal of Robotic systems 4 (5) (1987) 631-652.

[18] G. Weiss, M. Häfele, Repetitive control of mimo systems using $h^{\infty}$ design, Automatica 35 (7) (1999) 1185-1199.

[19] J. Ghosh, B. Paden, Nonlinear repetitive control, IEEE Transactions on Automatic Control 45 (5) (2000) 949-954.

[20] L. Paunonen, S. Pohjolainen, Internal model theory for distributed parameter systems, SIAM Journal on Control and Optimization 48 (7) (2010) 4753-4775.

[21] L. Paunonen, Robust controllers for regular linear systems with infinite-dimensional exosystems, SIAM Journal on Control and Optimization 55 (3) (2017) 1567-1597.

[22] D. Astolfi, L. Praly, L. Marconi, Approximate regulation for nonlinear systems in presence of periodic disturbances, in: 54th IEEE Conference on Decision and Control (CDC), 2015, pp. 7665-7670.

[23] D. Astolfi, L. Praly, L. Marconi, Francis-wonham nonlinear viewpoint in output regulation of minimum phase systems, IFAC-PapersOnLine 52 (16) (2019) 532-537.

[24] A. Serrani, A. Isidori, L. Marconi, Semi-global nonlinear output regulation with adaptive internal model, IEEE Transactions on Automatic Control 46 (8) (2001) 1178-1194.

[25] A. Isidori, Nonlinear control systems (1995).

[26] F. Mazenc, L. Praly, Adding integrations, saturated controls, and stabilization for feedforward systems, IEEE Transactions on Automatic Control 41 (11) (1996) 1559-1578.

[27] S. Marx, L. Brivadis, D. Astolfi, Forwarding design for stabilization of acoupled transport equation/ode with acone-bounded input nonlinearity, in: 59th IEEE Conference on Decision and Control, 2020.

[28] G. Bastin, J.-M. Coron, Stability and boundary stabilization of 1-d hyperbolic systems, Vol. 88, Springer, 2016.

[29] H. K. Khalil, Nonlinear systems, Prentice Hall, Third Edition, 2002

[30] T. Yoshizawa, Stability theory by liapunov's second method.

[31] M. Tucsnak, G. Weiss, Observation and control for operator semigroups, Springer, 2009

[32] R. Ortega, A. Astolfi, N. E. Barabanov, Nonlinear pi control of uncertain systems: an alternative to parameter adaptation, Systems \& control letters 47 (3) (2002) 259-278.

[33] L. C. Evans, Partial differential equations, 2nd Edition, Vol. 19 of Graduate Studies in Mathematics, American Mathematical Society, 2010

[34] M. Mattioni, S. Monaco, D. Normand-Cyrot, Forwarding stabilization in discrete time, Automatica 109 (2019) 108532.

[35] B. Wang, J. Wang, B. Zhang, X. Li, Global cooperative control framework for multiagent systems subject to actuator saturation with industrial applications, IEEE Transactions on Systems, Man, and Cybernetics: Systems 47 (7) (2016) 1270-1283.

[36] G. Casadei, D. Astolfi, Multipattern output consensus in networks of heterogeneous nonlinear agents with uncertain leader: A nonlinear regression approach, IEEE Transactions on Automatic Control 63 (8) (2017) 2581-2587.

[37] Y. Chitour, S. Marx, C. Prieur, $L^{p}$-asymptotic stability analysis of a $1 \mathrm{~d}$ wave equation with a nonlinear damping, Journal of Differential Equations 269 (10) (2020) 8107-8131.

[38] M. Slemrod, Feedback stabilization of a linear control system in Hilbert space with an a priori bounded control, Mathematics of Control, Signals and Systems 2(3) (1989) 847-857.

[39] S. Marx, V. Andrieu, C. Prieur, Cone-bounded feedback laws for $m$-dissipative operators on Hilbert spaces, Mathematics of Control, Signals and Systems 29 (4) (2017) 18. 